\title{
The synergism of natural antimicrobials, antimycotics and more than anything else antioxidants is the prime goal to achieve an harmonic wound healing, surgically treated.
}

\author{
Texia Research ${ }^{1}$, Lorenzo Martini ${ }^{2}$ \\ ${ }^{1}$ Texia Research-Eu-TURIN, ${ }^{2}$ Department of Pharmaceutical Biotechnologies University of Siena
}

\begin{abstract}
I have always sustained that is hypoxia, evoked by antioxidants of whichever nature or origin, the chief responsible of the starting of the slow process of wound healing, and so, contrarily to certain old $A A$ who assumed that peroxides (e.g. Hydrogen peroxide) were the best remedies to get a rapid cicatrisation, I oppose my theory that all substances fetching oxygen (as ROS) are useful for a decent wound healing, but they are always cause of the formation of keloids that persist perennially. When a wound must be cured surgically, too many are the factors to be considered, not only the presence of bacteria Gram positive and negative,(some of these are favourable to cicatrisation though) but many other modulators and fungal aggressions, that represent a real hazard and constitute a serious delay in harmonic cicatrisation processes. I have invented a complex medical device apt to treat surgical wound healing in a special way that drives to an harmonic restitutio ad integrum of the function laesa and the absolute hindrance of the formation of harsh and irreversible keloids. The pomade, that is a medical device, comprises antioxidant agents, antimicrobial principles, healing enhancers and antifungal actives at high percentages.
\end{abstract}

Keywords: ROS, wound healing, Troll and Cannan's method, skin grafting.

\section{Background}

Physiological wound healing is a dynamic stepwise process consisting of partially overlapping phases that are determined by interacting events on a molecular, cellular and extracellular matrix level. This process, which is not yet clear, starts with a disturbance of tissue integrity and ends with a restitutio ad integrum or a scar formation within an appropriate length of time. Only the first step of wound healing requires oxygen and it is hypoxia the fundamental starter to trigger the ulterior steps of the whole process. Three physiological phases must be distinguished: the inflammatory phase, the proliferative phase (that forecasts neoangiogenesis, tissue formation, re-epithelialization) and the tissue remodelling phase.

The inflammatory phase lasts between 4 and 6 days and starts immediately after wounding. Blood vessels constrict after traumatization, and platelets aggregate along the activated endothelium. Vascular disruption and vasoconstriction cause a hypoxic microenvironment that is intensified by increased oxygen consumption due to metabolically active cells contributing to wound healing. Hypoxia actuates the initial step of wound healing by boosting ROS activity, by activating platelets and endothelium, and by inducing cytokines released from platelets, monocytes and parenchymal cells [e.g. vascular endothelial growth factor (VEGF).

I have always sustained that is hypoxia, evoked by antioxidants of whichever nature or origin, is the chief responsible of the starting of the slow process of wound healing, (almost in the second and third phases) and so, contrarily to old AA who assumed that peroxides (e.g. Hydrogen peroxide) were the best remedies to get a rapid cicatrisation, I oppose my theory that all substances fetching oxygen are useful for a decent wound healing, but are always cause of the formation of keloids that persist perennially.

When a wound must be cured surgically, too many are the factors to be considered, not only the presence of bacteria Gram positive and negative, but many other modulators and fungal aggressions.

I have invented a complex medical device apt to treat surgical wound healing in a special way that drives to an harmonic restitution ad integrum of the function laesa and the absolute hindrance of the formation of harsh and irreversible keloids.

As far as the Proliferative Phase is concerned - depending on the extent of the wound - for a few weeks and comprises elementary processes such as neovascularization, formation of granulation tissue and ECM, and re-epithelialization. Endothelial cells and fibroblasts simultaneously invade the initially built haemostatic clot. Macrophages lead the way by degrading the clot and by releasing cytokines and chemokines that attract fibroblasts and stimulate angiogenesis. Particularly macrophages and their metabolites play a pivotal role in granulation tissue formation as depletion of macrophages was shown to lead to impaired wound healing in an in vivo porcine model.Fibroblasts and keratinocytes also secrete growth factors. Hereby, the cytokines of the TGF- $\beta$ superfamily seem to play the most prominent role in granulation tissue formation. Interestingly, ECM molecules such as fibrinogen, fibronectin, fibrin and vitronectin are interactive with cytokines and also 
The synergism of natural antimicrobials, antimycotics and more than anything else antioxidants is..

regulate the proliferation, differentiation and migration of fibroblasts. Important stimulators of angiogenesis are hypoxia and ROS. Both stimulate macrophages, fibroblasts, endothelial cells and keratinocytes to synthesize VEGF. Even in the proliferative phase, acute hypoxia is the initiator of this process, whereas chronic hypoxia impairs neovascularization.Hypoxia activates the transcription factor hypoxia-inducible factor (HIF)- $1 \alpha$. HIF-1 $\alpha$ binds to the hypoxia response element in the gene promoter region of the VEGF gene, which in turn upregulates VEGF. VEGF, as the major angiogenic growth factor, stimulates endothelial cells to migrate, proliferate and form countless new capillaries. The new capillaries branch out and invade the provisional wound matrix, which is replaced piecemeal by a new ECM produced and deposited by fibroblasts. The emerging ECM, in which fibroblasts, myofibroblasts, leucocytes and macrophages are embedded, consists of immature collagen (type III), proteoglycans, glycosaminglycans, fibrin, fibronectin and hyaluronic acid.In this context, the production and deposition of collagen represents a fundamental process as it reconstitutes skin alignment and integrity. The production and deposition of collagen is proportional to oxygen tension: fibroblasts need a $\mathrm{pO}_{2}$ of $30-40 \mathrm{mmHg}$ for collagen synthesis. A central oxygen-dependent step in the synthesis of collagen is the hydroxylation of proline and lysine residues. In addition, hydroxylase activity is critically dependent on cofactors such as iron and vitamin C. Lysyl hydroxylase and lysyl oxidase, both oxygen-dependent enzymes, catalyse collagen crosslinking, a step that aims at wound stability. Again, in hypoxia, acute hypoxic conditions must be distinguished from chronic hypoxia. Acute hypoxia may stimulate fibroblast proliferation, collagen synthesis and expression of TGF- $\beta 1$, whereas chronic hypoxia decreases these processes as shown in vitro by Siddiqui et al. in human dermal fibroblasts. Angiogenesis and ECM synthesis are interdependent processes as new blood vessels need new ECM as a three-dimensional scaffold for their ingrowth while the cell metabolism of, for example, fibroblasts needs new blood vessels that deliver oxygen and other nutrients. The fact that the same cytokines stimulate each process interconnects these steps of wound healing. Parallel to the formation of granulation tissue, re-epithelialization is initiated.(1-6)

Also the fungal aggression during the surgical treatment of a wound has to be seriously considered in order to avoid their radication that can compromise an harmonic healing with misleading formation of keloids.

Effectively Surgical infections are infections of the wounds evoked thanks to the surgical procedure. The development of these types of infections is related to 3 factors; the degree of microbial contamination of the wound during surgery, the duration of the procedure and host factors, such as diabetes, malnutrition, obesity, immunosuppression, and the number of other underlying disease states.

Bacteria are responsible for the majority of surgical infections. Gram-positive bacteria that frequently cause infections in surgical patients include aerobic skin commensals and enteric organisms. There are many pathogenic gram-negative bacterial species that are capable of causing surgical infections, predominantly members of the Enterobacteriaceae family. Anaerobic bacteria, fungi, and viruses are the other causes of SIs. Fungi cause nosocomial infections in surgical patients as a part of polymicrobial infections or fungemia, rare causes of aggressive soft tissue infections and so-called opportunistic pathogens.

In this study we want to deep the capacity of a medical device (rich in antioxidants, antifungal principles, antimicrobial agentsand healing enhancers) to treat especially wounds evoked by skin grafts and sutures.

Skin grafting involves taking a piece of healthy skin from one part of the body and using it to repair another area of the body. Aesthetic surgeons use skin grafting to cover and close wounds where there is a lack of adjacent skin to use. The transplanted healthy skin heals into the new location. Skin grafts are often used to help burn victims.

All sutures are classified as either absorbable or non-absorbable depending on whether the body will naturally degrade and absorb the suture material over time. Absorbable suture materials include the original catgut as well as the newer synthetics polyglycolic acid, polylactic acid, polydioxanone, and caprolactone. They are broken down by various processes including hydrolysis (polyglycolic acid) and proteolytic enzymatic degradation. Depending on the material, the process can be from ten days to eight weeks. They are used in patients who cannot return for suture removal, or in internal body tissues. ${ }^{\text {[7] }}$ In both cases, they will hold the body tissues together long enough to allow healing, but will disintegrate so that they do not leave foreign material or require further procedures. Occasionally, absorbable sutures can cause inflammation and be rejected by the body rather than absorbed.

Non-absorbable sutures are made of special silk or the synthetics polypropylene, polyester or nylon. Stainless steel wires are commonly used in orthopedic surgery and for sternal closure in cardiac surgery. These may or may not have coatings to enhance their performance characteristics. Non-absorbable sutures are used either on skin wound closure, where the sutures can be removed after a few weeks, or in stressful internal environments where absorbable sutures will not suffice. Examples include the heart (with its constant pressure and movement) or the bladder (with adverse chemical conditions). Non-absorbable sutures often cause less scarring because they provoke less immune response, and thus are used where cosmetic outcome is important. They may be removed after a certain time, or left permanently. 
The synergism of natural antimicrobials, antimycotics and more than anything else antioxidants is..

Our attention was focused on the repair and the restitutio ad integrum of wounds evoked by skin grafts and sutures, after removal, especially the non-absorbable ones.

\section{Materials and methods}

The pomade contains 6 antioxidant agents (sodium citrate, copper sulphate, manganese sulphate, manganese gluconate, Salvia off. Oil, Rosmarinus off. Oil), 7 antimicrobial agents (Zinc sulphate, chlorexidine digluconate,chloroxylenol, benzalkonium chloride and Salvia and Rosmarinus off. Oils but even Thymus vulgaris oil), 4 healing enhancers (idest bisabolol, asiaticoside from Centella asatica, Pegaga sp., Sodium hyaluronate and Melia azadirachta seed oil) and finally two important and drastic antifungal actives ( idest Clotrimazole and Ciclopirox Olamine).

We have recruited three individuals who willingly decided to try our medical device:

1) A man (41 y.old) who underwent a nasal septum reconstruction

2) A woman (35 y. old) who underwent a lips reconstruction by skin grafting

3) A man (66 y.old) who underwent a sternal closure after a cardiac intervention ( for the suture was employed polypropelene thread).

The pomade (idest the medical device) has been spread twice a day for 6 days in each of every case.

It is important and noticeable to remark that the treatment with the medical device began only after five days after the thread removal, and the medical cure, in the first phase, that corresponds to the inflammatory step, was carried out only by washing with $10 \%$ hydrogen peroxide solution and spreading topical powder containing neomycin sulphate, three times a day.

The evaluation of the coefficient of the complete wound healing (by means of a complete and harmonic skin reconstruction) has been realized following the Troll and Cannan's method

(7-11) based principally on the determination of the ratio proline/ hydroxyproline in samples of scar tissues.(that is the crust of a wound, detached with a scalpel during the proliferative phase of the cicatrisation).

These authors have observed that proline and hydroxyproline react with ninhydrin in two steps. Ordinarily, a transient red pigment is first formed which is converted rapidly to a stable yellow pigment. However, they have established conditions under which the red pigment may be completely extracted with benzene from the reaction system and thus stabilized for calorimetric measurement. The red pigment derived from the reaction of proline with ninhydrin exhibits an absorption spectrum with a maximum at a wave-length of $550 \mathrm{rnp}$; that from hydroxyproline, a maximum at $570 \mathrm{rnp}$. A comparison of the absorptions at each wavelength permits the separate determination of these two amino acids in a mixture containing substantially equivalent amounts of each. This is the method described by Troll and Cannan for the spectrophotometric determination of praline and of hydroxyproline after development of the specific ninhydrin color investigation.

However, in hydrolysates of scar tissue traces of proline (deriving from collagen) and htdroxyprolyne (deriving from plasma) represent the index of the presence of both the aminoacids: the proline represents the quantity of naïve collagen (index of quasi-complete cicatrisation) meanwhile hydroxyprolyne represents the concentration of plasma, that indicates the incomplete or "in fieri" cicatrisation.

A good and harmonic wound healing should be represented by a ratio where the proline content must be inevitably higher than that of hydroxyproline because of the co-presence of the plasma itself.

The scale scores goes from nil to 1 ., where values proximal to nil indicate that cicatrisation is not complete at all, since the presence of prolyne (index of the presence of collagen) is overwhelming, with regards to hydroxyproline.

\section{Results}

In Case 1 the scar tissue was detached at he fourth day and the score recorded for the hydroxyproline analysis was 1 .

In Case 2 the scar tissue was detached at he fifth day and the score recorded for the hydroxyproline analysis was 1 .

In case 3 the scar tissue was detached at he sixth day and the score recorded for the hydroxyproline analysis was 0.79 .

\section{Conclusions}

These scores are undoubtedly encouraging.

In Case 3, surgeons assert that the wound was deeper and x-rays showed a severe injury of the stratum granulosum, during the surgical procedure and so, there were the evidence of a slight presence of plasma though. 


\section{References}

[1]. Neuman, R. E., and Logan, M. A., J. Biol. Chem., 184,299 (1950).

[2]. McFarlane, W. D., and Guest, G. H., Canad. J. Res., Sect. B, 17, 139 (1939)

[3]. Campbell, D. H., Koepfli, J. B., Pauling, L., Abrahamsen, N., Dandliker, W., Feigen, G. A,, Lanni, F., and LeRosen, A., Texas Rep. Biol. and Med., 9, 235 (1951).

[4]. Higgins, A. R., Harper, H. A., Kimmel, J. R., Burns, T. W., Jones, R. E., Smith, T. W. D., and Klein, C. J., J. Appl. Physiol., 4,776 (1952).

[5]. Troll, W., and Cannan, R. K., J. Biol. Chem., 200,803 (1953).

[6]. Moore, S., and Stein, W. H., J. Biol. Chem., 192,663 (1951). Page 10 of 10

[7]. Graham KM, Singh R, Millman G, Malnassy G, Gatti F, Bruemmer K, Stefanski C, Curtis H, Sesti J, Carlson CG. (2010). Excessive collagen accumulation in dystrophic ( $\mathrm{mdx}$ ) respiratory musculature is independent of enhanced activation of the NFkappaB pathway. J Neurol Sci. 294(1-2), 43-50

[8]. Prockop D.J. and Udenfriend S., (1960). A specific method for the analysis of hydroxyproline in tissues and urine. Analytical Biochemistry, 1, 228-239.

[9]. Stedman, H.H., Sweeney, H.L., Shrager, J.B., Maguire, H.C., Panettieri, R.A., Petrof, B., Narusawa, M., Leferovich, J.M., Sladky, J.T., Kelly, A.M., (1991). The mdx mouse diaphragm reproduces the degenerative changes of Duchenne muscular dystrophy. Nature 352, 536- 539.

[10]. Switzer, B.R., Summer, G.K., (1971). Improved method for hydroxyproline analysis in tissue hydrolyzates. Anal Biochem. 39(2), 487-491.

[11]. Rogers CJ, Kimmel JR,Hutchin ME,Harpers AA: A hydroxyprolyne method of analysis for a gelatine in plasama and urine. From the Metabolic Research Facility, United States Naval Hospital, Oakland, California) (Received for publication, July 22, 1953) 